SUBJECT AREAS:

STRUCTURE OF SOLIDS

AND LIQUIDS

INFORMATION STORAGE

Received

20 August 2014

Accepted

31 December 2014

Published

30 January 2015

Correspondence and requests for materials should be addressed to E.M.V. (vmadhavan@ qf.org.qa)

\title{
Structural transition and enhanced phase transition properties of Se doped $\mathrm{Ge}_{2} \mathrm{Sb}_{2} \mathrm{Te}_{5}$ alloys
}

\author{
E. M. Vinod', K. Ramesh ${ }^{2}$ \& K. S. Sangunni ${ }^{2}$
}

\author{
'Qatar Environment and Energy Research Institute, P.O. Box: 5825, Doha, Qatar, ${ }^{2}$ Department of Physics, Indian Institute of \\ Science, Bangalore, India-560012.
}

Amorphous $\mathrm{Ge}_{2} \mathrm{Sb}_{2} \mathrm{Te}_{5}$ (GST) alloy, upon heating crystallize to a metastable $\mathrm{NaCl}$ structure around $150^{\circ} \mathrm{C}$ and then to a stable hexagonal structure at high temperatures $\left(\geq 250^{\circ} \mathrm{C}\right)$. It has been generally understood that the phase change takes place between amorphous and the metastable $\mathrm{NaCl}$ structure and not between the amorphous and the stable hexagonal phase. In the present work, it is observed that the thermally evaporated $(\mathrm{GST})_{1-\mathrm{x}} \mathrm{Se}_{\mathrm{x}}$ thin films $(0 \leq \mathrm{x} \leq 0.50)$ crystallize directly to the stable hexagonal structure for $\mathrm{x} \geq$ 0.10 , when annealed at temperatures $\geq 150^{\circ} \mathrm{C}$. The intermediate $\mathrm{NaCl}$ structure has been observed only for $\mathrm{x}<\mathbf{0 . 1 0}$. Chemically ordered network of GST is largely modified for $\mathrm{x} \geq 0.10$. Resistance, thermal stability and threshold voltage of the films are found to increase with the increase of Se. The contrast in electrical resistivity between the amorphous and crystalline phases is about 6 orders of magnitude. The increase in Se shifts the absorption edge to lower wavelength and the band gap widens from 0.63 to $1.05 \mathrm{eV}$. Higher resistance ratio, higher crystallization temperature, direct transition to the stable phase indicate that $(\mathrm{GST})_{1-\mathrm{x}} \mathrm{Se}_{\mathrm{x}}$ films are better candidates for phase change memory applications.

$\mathrm{t}$ is well known that $\mathrm{Ge}_{2} \mathrm{Sb}_{2} \mathrm{Te}_{5}$ (GST) alloy undergo reversible phase transition between the amorphous and crystalline states by laser irradiation or electrical pulses ${ }^{1-2}$. Upon applying an electric field, low conductive amorphous state crosses a threshold voltage $\left(\mathrm{V}_{\mathrm{th}}\right)$ and permanently changes to a high conductive crystalline state due to the Joule heating (SET transition). To revert back to the amorphous state, a nano second pulse with very low rise and fall time is applied. This increases the temperature of the crystalline GST above its melting point $\left(\mathrm{T}_{\mathrm{m}}\right)$ and then the material rapidly cooled back to room temperature which happens within 10-30 ns (RESET transition). The amorphous and crystalline phases have large resistance contrast, at least a minimum of $10^{3}$. This property of GST alloys is exploited for the phase change random access memory (PCRAM) applications.

The rate of crystallization, thermal stability and the contrast in electrical resistivity and optical reflectivity are very important for an efficient phase change material. In $\mathrm{Ge}-\mathrm{Sb}-\mathrm{Te}$ along the $\mathrm{Sb}_{2} \mathrm{Te}_{3}-\mathrm{GeTe}$ pseudo binary tie-line the thermal stability increases but the crystallization speed decrease. At the extreme ends of this tie-line, GeTe has higher thermal stability and less crystallization speed while $\mathrm{Sb}_{2} \mathrm{Te}_{3}$ has higher crystallization speed and lower thermal stability. $\mathrm{Ge}_{2} \mathrm{Sb}_{2} \mathrm{Te}_{5}$ is an intermediate compound in the tie-line and so has a best combination of thermal stability and crystallization speed. However, the low thermal stability and the requirement of higher RESET current due to higher $\mathrm{T}_{\mathrm{m}}\left(616^{\circ} \mathrm{C}\right)$ are some of the concerns ${ }^{3-4}$.

Another aspect is the toxicity of Te due to which Te is less preferred for semiconductor manufacturing processes. Selenium is less toxic than Te, hence a constant effort is being made to use more of Se in place of Te in the phase change memory (PCM) alloys ${ }^{3}$. An increase in electrical resistivity and a decrease in crystallization temperature $\left(\mathrm{T}_{\mathrm{c}}\right)$ has been observed in $\mathrm{GeSb}_{2} \mathrm{Te}_{4}$ when $\mathrm{Se}$ is substituted for $\mathrm{Te}^{3}$. $\mathrm{GeSb}_{2} \mathrm{Te}_{3} \mathrm{Se}$ shows usual amorphous to $\mathrm{NaCl}$ and then to hexagonal transition even after 25 at.\% of Te sites are substituted by Se. In the rock salt structure of GST, $20 \%$ of vacancy is reported in Ge/Sb sublattice, whereas hexagonal structure has none ${ }^{4}$. Other $\mathrm{Se}$ alloys such as $\mathrm{Ga}-\mathrm{Sb}-\mathrm{Se}$, Sb-Se possess higher $\mathrm{T}_{\mathrm{c}}$, better data retention, higher switching speed, lower thermal conductivity and lower $\mathrm{T}_{\mathrm{m}}$ with respect to GST, but the resistance ratio is limited to about two orders of magnitude ${ }^{5-6}$. If a comparable resistance ratio along with a higher $\mathrm{T}_{\mathrm{c}}$ can be obtained by doping GST with a suitable material, it would be beneficial. In this work, an attempt has been made to improve the phase change properties of GST by the addition of Se for PCM applications. 


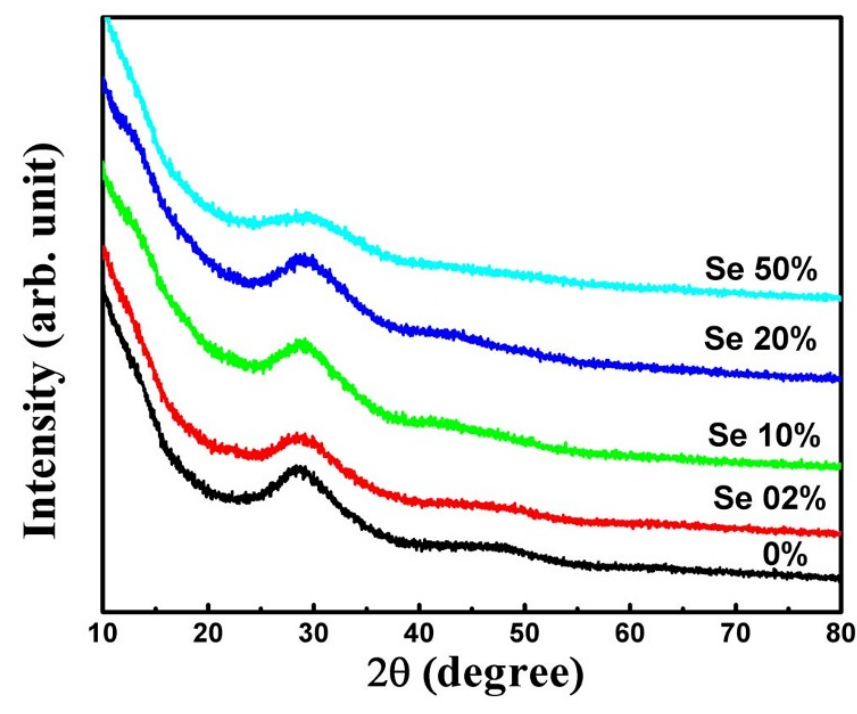

Figure $1 \mid$ XRD pattern of $(\mathrm{GST})_{1-} \mathrm{Se}_{\mathrm{x}}$ films in the as deposited state.

\section{Results and Discussion}

The XRD spectra shown in Fig. 1 indicate the amorphous nature of the as deposited (AD) $(\mathrm{GST})_{1-\mathrm{x}} \mathrm{Se}_{\mathrm{x}}$ films. The films annealed at $150^{\circ} \mathrm{C}$ and $300^{\circ} \mathrm{C}$ are shown in Fig. 2. The crystalline structure of GST and $(\mathrm{GST})_{0.98} \mathrm{Se}_{0.02}$ films annealed at $150^{\circ} \mathrm{C}$ are identified as $\mathrm{NaCl}$ with space group Fm-3m (Fig. 2a) (04-011-9024 ICDD 2012) ${ }^{7}$. Interestingly the films with $\mathrm{x}=0.10$ and 0.20 crystallized into hexagonal structure which was not expected (04-006-9784 ICDD 2012) ${ }^{8}$. The peak intensity of these alloys decreases with the increase of Se indicating increased amorphousness in the alloys. The presence of Se may suppress the crystallization of GST and therefore a very small percentage of GST nucleates with small crystallite size. The decrease in peak intensity and broadening of the peaks indicate a decrease in grain size for Se doped GST films. Similar kind of decrease in grain size has been observed in Ag doped GST ${ }^{9}$. The $(\mathrm{GST})_{0.50} \mathrm{Se}_{0.50}$ film remain amorphous and does not crystallize. The films annealed at $300^{\circ} \mathrm{C}$ crystallized into hexagonal structure as shown in Fig. 2(b).

It is interesting to note that the intermediate $\mathrm{NaCl}$ phase disappeared with the Se doping. Atomic distribution of GST in crystalline $\mathrm{NaCl}$ structure closely resembles that of its amorphous phase ${ }^{10}$. This necessitates only a small change in local structure during the phase transformation. Kolobov et al. suggest that the coordination of Ge atoms changes from octahedral to tetrahedral co-ordination (Umbrella flip) during the phase change ${ }^{11}$. There is Ge-Te, Sb-Te bonds in the first neighbor interactions and $\mathrm{Te}-\mathrm{Te}$ bonds in the second neighbor interactions in both amorphous and crystalline
Table 1 Atomic weight, atomic radius and electronegativity of $\mathrm{Ge}, \mathrm{Sb}, \mathrm{Te}$ and Se elements

\begin{tabular}{lcccc} 
& $\mathrm{Ge}$ & $\mathrm{Sb}$ & $\mathrm{Te}$ & $\mathrm{Se}$ \\
\hline At wt.(amu) & 72.64 & 121.76 & 127.60 & 78.96 \\
At radius (pm) & 125 & 133 & 123 & 103 \\
Electronegativity (Pauling scale) & 2.01 & 2.05 & 2.1 & 2.55 \\
\hline
\end{tabular}

phases ${ }^{11}$. Amorphous phase has bonded with natural valency followed by $8-\mathrm{N}$ rule and $\mathrm{NaCl}$ structure has 6 fold co-ordinations. In $\mathrm{NaCl}$ structure of GST, the $4 \mathrm{a}$ site is wholly occupied by Te and the $4 \mathrm{~b}$ site is randomly occupied by $\mathrm{Ge}$ or $\mathrm{Sb}$ or $20 \%$ vacancies $^{10}$. The transition from $\mathrm{NaCl}$ to hexagonal happens with minimum changes and with the conservation of bond lengths ${ }^{12}$.

Zhimei Sun et al. ${ }^{12}$ discussed that the metastable GST does not possess a cubic structure, rather consists of two well-defined highly ordered three-dimensional repeat units: $-\mathrm{Te}-\mathrm{Ge}-\mathrm{Te}-\mathrm{Sb}-\mathrm{Te}-(\mathrm{I})$ and -Te-Sb-Te-Ge- (II). These two units repeat and are bonded alternatively in space consistent with a $\mathrm{NaCl}$ structure. The phase transition from $\mathrm{NaCl}$ to hexagonal is made possible by the movement of unit II in the [210] direction. And there exist a weak bonding between Te layer of each unit to others and phase change is possible by the movement of Te planes ${ }^{12}$.

Upon heating, GST films crystallize to $\mathrm{NaCl}$ structure and then to hexagonal structure at $150^{\circ} \mathrm{C}$ and $250^{\circ} \mathrm{C}$ respectively ${ }^{13}$. Ag doped GST show direct amorphous to $\mathrm{NaCl}$ transition and no indication of hexagonal phase transition in the studied temperature range (up to $\left.300^{\circ} \mathrm{C}\right)^{9}$. This study ruled out the possibility of Ag occupancy in the vacancies but suggest the possibility of $\mathrm{Ag}$ atoms being occupied at the grain boundaries. Atomic radius of $\mathrm{Ag}$ is $165 \mathrm{pm}$ and atomic weight is $107.9 \mathrm{amu}$, which can be compared with the atoms present in the GST alloy (Table 1). It was found that in $\mathrm{Ge}_{2} \mathrm{Sb}_{2+\mathrm{x}} \mathrm{Te}_{5}$ alloys, $\mathrm{Sb}$ atoms added beyond the stoichiometric ratio do not enter the lattice site of the $\mathrm{NaCl}$ type crystal to fill up the vacancies but remain at the grain boundaries and suppresses the crystal growth ${ }^{10}$. Phase separation has been observed in the non-stoichiometric alloys rich with $\mathrm{Ge}^{14}$. Indium doped GST $\left(\mathrm{In}_{0.3} \mathrm{Ge}_{2} \mathrm{Sb}_{2} \mathrm{Te}_{5}\right)$ show a mixed phase of dominant cubic structure and a small amount of $\mathrm{In}_{2} \mathrm{Te}_{3}$ when annealed at $180^{\circ} \mathrm{C}^{15}$. Amorphous to cubic transition was observed above $220^{\circ} \mathrm{C}$ in $\mathrm{Si}$ doped GST for $\mathrm{Si} \geq 11.8$ at. \% whereas the $\mathrm{Si}<$ 11.8 at.\% shows amorphous to $\mathrm{NaCl}$ and then to hexagonal transitions above $170^{\circ} \mathrm{C}$. The smaller atomic size and atomic weight and higher electronegativity of Se influence the structural transitions in GST.

In chalcogenides, the variations of physical properties with composition are well understood with the constraint counting model
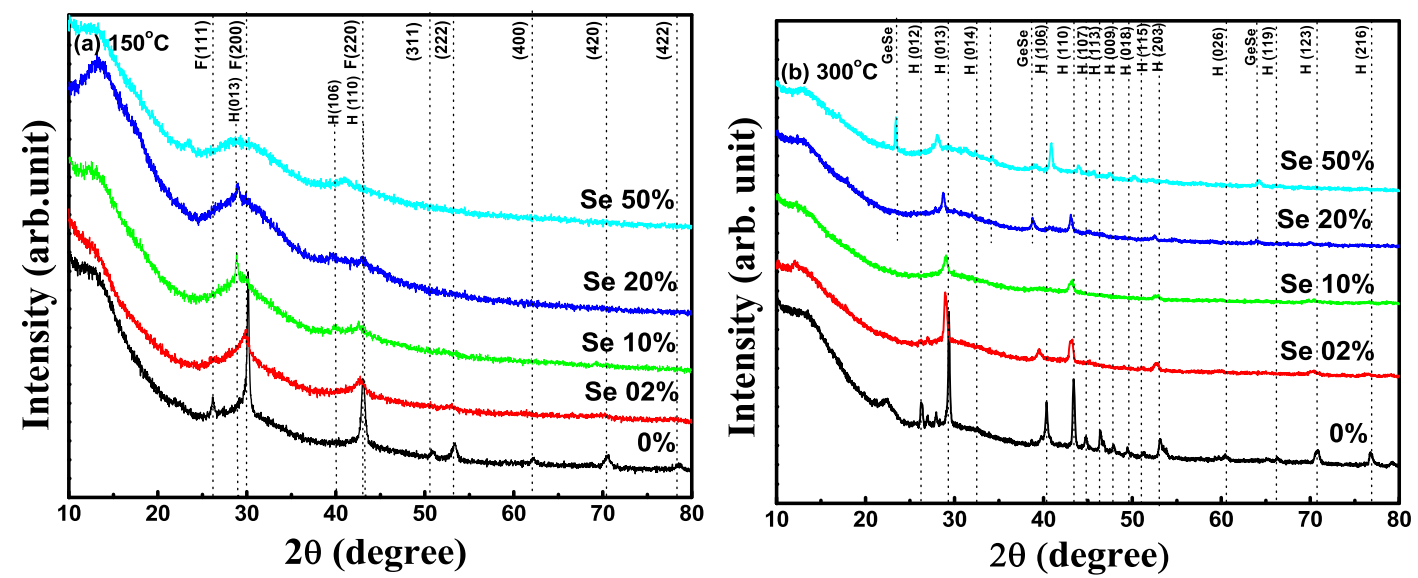

Figure $2 \mid \mathrm{XRD}$ patterns of (GST) ${ }_{1-\mathrm{x}} \mathrm{Se}_{\mathrm{x}}$ films annealed at (a) $150^{\circ} \mathrm{C}$ and (b) $300^{\circ} \mathrm{C}$. 
Table 2 | Average co-ordination number of the alloys

$\left(\mathrm{Ge}_{2} \mathrm{Sb}_{2} \mathrm{Te}_{5}\right)_{1-\mathrm{x}} \mathrm{Se}_{\mathrm{x}} \quad \mathrm{x}=0.0 \quad \mathrm{x}=0.02 \quad \mathrm{x}=0.10 \quad \mathrm{x}=0.20 \quad \mathrm{x}=0.50$

\begin{tabular}{llllll}
\hline Co-ord. no. & 2.67 & 2.65 & 2.6 & 2.53 & 2.33
\end{tabular}

proposed by Phillips and Thorpe ${ }^{16}$. According to this model the network transform from floppy (under-coordinated network) to a rigid structure (over-coordinated network) at an average coordination $\mathrm{Z}_{\mathrm{av}}=2.4$. The critical composition at which this transition occurs is called the rigidity percolation threshold (RPT). There is another transition called chemical threshold (CT) which usually occurs at higher coordination numbers. In IV-VI glasses CT occurs at $\mathrm{Z}_{\mathrm{av}}=2.67$ and for $\mathrm{V}-\mathrm{VI}$ glasses both the RPT and CT coincides at $\mathrm{Z}_{\mathrm{av}}=2.4$. At CT, the network has a maximum ordering and is closest to the crystalline state ${ }^{17}$. The molar volume is also exhibits a maximum at CT. Interestingly; the average co-ordination number of GST is 2.67 , which corresponds to the chemically ordered network structure. This chemical ordering could be one of the reasons for the ease of transition between amorphous and crystalline states in GST alloy. Addition of Se to GST, decreases the average coordination number (see Table 2). The decrease in average coordination number indicates the decrease in the network rigidity, molar volume and deviation from chemical ordering. Hence, with the increase of Se, crystallization may become difficult. There should be a critical composition at which this decrease in average coordination, molar volume, rigidity and chemical ordering should largely affect the crystallization and thereby the phase change properties. In the (GST) ${ }_{1-\mathrm{x}} \mathrm{Se}_{\mathrm{x}}$ alloys this critical coordination occurs at $x \geq 0.10\left(Z_{a v}=2.6\right)$ where the intermediate $\mathrm{NaCl}$ phase disappears and the alloy directly undergo amorphous to hexagonal transition.

Out of the three well known phases of GST, the stable hexagonal phase consists of cycling nine layers of $-\mathrm{Te}-\mathrm{Ge} / \mathrm{Sb}-\mathrm{Te}-\mathrm{Ge} / \mathrm{Sb}-\mathrm{Te}-$ $\mathrm{Te}-\mathrm{Ge} / \mathrm{Sb}-\mathrm{Te}-\mathrm{Ge} / \mathrm{Sb}$ - in the $\mathrm{ABCABCABC}$ packing in one unit cell (space group $\mathrm{P}-3 \mathrm{ml}$ with lattice parameter $\mathrm{a}=4.2 \AA, \mathrm{c}=16.96 \AA)^{18}$. Metastable $\mathrm{NaCl}$ structure can also be considered as six layered structure, - $\mathrm{Te}-\mathrm{Ge} / \mathrm{Sb}-\mathrm{Te}-\mathrm{Ge} / \mathrm{Sb}-\mathrm{Te}-\mathrm{Ge} / \mathrm{Sb}-$, in the hexagonal notation in which alternate stacks of $\mathrm{Te}$ and $\mathrm{Ge} / \mathrm{Sb}$ layer are cycled along c-axis ${ }^{4}$. This leads to a conclusion that the stable hexagonal phase can be considered as $\mathrm{NaCl}$ layers stacked along the c-axis forming nine layers, except the Te-Te layer. Considering metastable structure having vacancy (20 at.\%) and stable phase having a Te-Te layer (without vacancy), it would be possible that Se occupies the rock salt structure vacancies and forms an Te-Te or Se-Te van der Waals bonded layers, and can possibly leads to a hexagonal structure. The above models are based on the fact that both metastable and stable phases are fundamentally similar to each other, when Te and Ge/Sb stacked alternatively. However, these assumptions need a detailed study. The general understanding is that the metastable NaCl-like structure is important for crystallization process in phase change GST alloys ${ }^{19}$. The amorphous to hexagonal transition in the Se doped GST alloys also results in the reversible switching with comparable threshold voltages.

Raman spectroscopy can be used to probe medium range order in glasses and crystalline materials. It is a very sensitive tool for studying PCM materials since photons can directly couple with lattice vibrations and inherently reflect the local symmetry. It is important to use appropriate measurement time and low power density laser beam in order to avoid laser induced crystallization of GST film. The accumulation time of the measurement is fixed to 180 seconds for varying power densities. Power of the laser can be reduced by using various filters. Spectra of GST AD films for varying power densities are shown in Fig. 3.

Raman bands centered at 110 and $165 \mathrm{~cm}^{-1}$ seen in Fig. 3(a-d) are characteristic of crystalline GST, signifying the laser induced crystallization in the sample. Cho et al. and Liu et al. ${ }^{20-21}$ observed Raman

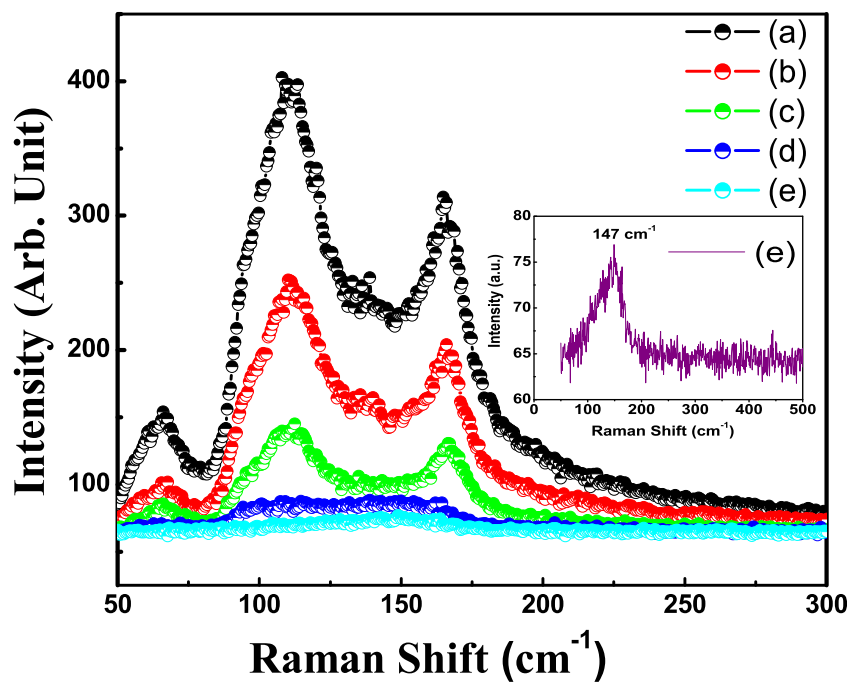

Figure 3 The spectra of GST for various filter-power combinations. (a) $3 \mathrm{~mW}$ laser power without filter, (b) $1 \mathrm{~mW}$ without filter, (c) $1 \mathrm{~mW}$ with D0.3, (d) $1 \mathrm{~mW}$ with D1, (e) $1 \mathrm{~mW}$ with D2. Inset shows the enlarge spectra of 3(e) resulting from the amorphous GST.

peaks for c-GST at $110 \mathrm{~cm}^{-1}$ corresponding to the vibration of hetero polar bond in the tetrahedral $\mathrm{GeTe}_{4}$ and/or pyramidal $\mathrm{SbTe}_{3}$. The band at $160 \mathrm{~cm}^{-1}$ is due to $\mathrm{Sb}-\mathrm{Sb}$ vibrations in $\left(\mathrm{Te}_{2}\right) \mathrm{Sb}-\mathrm{Sb}\left(\mathrm{Te}_{2}\right)$ or (TeSb)Sb-Sb( $\left.\mathrm{Te}_{2}\right)^{11,20-24}$ In Fig. 3(e) and the inset, a band centered at $147 \mathrm{~cm}^{-1}$ has been observed which originates from the amorphous GST. This band is believed to originate from $\mathrm{SbTe}_{3}$ pyramids or edge sharing $\mathrm{GeTe}_{4}$ tetrahedral vibrational modes ${ }^{11,19-20}$. As the power of the laser decreases or filtering increases, the surface crystallization disappears.

Fig. 4 show Raman spectra of AD and annealed (GST) ${ }_{1-\mathrm{x}} \mathrm{Se}_{\mathrm{x}}$ films. Amorphous GST has a Raman band centered at $147 \mathrm{~cm}^{-1}$. The Raman spectrum of a-GST and a-Sb $\mathrm{Te}_{3}$ is very similar ${ }^{25}$. For films annealed at $300^{\circ} \mathrm{C}$, peaks are observed at around 114 and $171 \mathrm{~cm}^{-1}$ (Fig. 4(b)). The peak observed at $171 \mathrm{~cm}^{-1}$ is due to the hexagonal structure. A peak at $160 \mathrm{~cm}^{-1}$ for the $\mathrm{NaCl}$ structure gets shifted to higher wave numbers in the hexagonal structure as reported by Bastiani et al. ${ }^{26}$ When atomic weight of the doped elements is less, the peak position shifts to higher wave number ${ }^{27}$. The peak shift and broadening indicate the change in the bond length and bond angles ${ }^{27}$. The band at 190-192 $\mathrm{cm}^{-1}$ is observed in both AD and annealed thin films of $(\mathrm{GST})_{0.50} \mathrm{Se}_{0.50}$. For $\mathrm{GeSe}_{4}$ tetrahedra vibrations, Raman bands are observed at 200 and $215 \mathrm{~cm}^{-128-29}$. Hence, the peaks observed at 192 and $210 \mathrm{~cm}^{-1}$ must be originated from the $\mathrm{GeSe}_{4}$ tetrahedra vibrations, but the shift in values may be due to the presence of crystalline phase formed in the GST. The Raman spectra of the Ge, Te and Se crystalline bulk elements were recorded to check the presence of individual elements phase, if any, in the AD and annealed films, but were absent ${ }^{30}$.

The near infrared transmittance spectra of $(\mathrm{GST})_{1-\mathrm{x}} \mathrm{Se}_{\mathrm{x}}$ thin films are shown in Fig. 5. Transmittance minimum decreases with increase of Se at.\% indicating the increase of band width. The spectral contribution of the absorption coefficient $(\alpha)$ was determined using the relation, $\alpha=(1 / \mathrm{d}) \ln \left(\mathrm{I}_{0} / \mathrm{I}\right)$ where $(\mathrm{Io} / \mathrm{I})$ is the transmittance and $\mathrm{d}$ is the film thickness. For higher values of $\left(\alpha>10^{4} \mathrm{~cm}^{-1}\right)$, the absorption coefficient $(\alpha)$ yields the power part which obeys the Tauc ${ }^{31}$ and Davis and Mott relation ${ }^{32}$ for the allowed indirect transition:

$$
(\alpha \mathrm{h} v)^{1 / 2}=\mathrm{B}^{1 / 2}\left(\mathrm{~h} v-\mathrm{E}_{\mathrm{opt}}\right)
$$

where $\mathrm{B}^{1 / 2}$ is the Tauc parameter, $\mathrm{h}$ is the Plank's constant, $v$ is the frequency, $\mathrm{E}_{\mathrm{opt}}$ is the optical band gap. $\mathrm{B}^{1 / 2}$ refers slope in the extended region and it is the measure of disorder ${ }^{33}$. Calculated $\mathrm{E}_{\mathrm{opt}}$ and $\mathrm{B}^{1 / 2}$ of GST and (GST) ${ }_{0.90} \mathrm{Se}_{0.10}$ films are given in Table 3. 

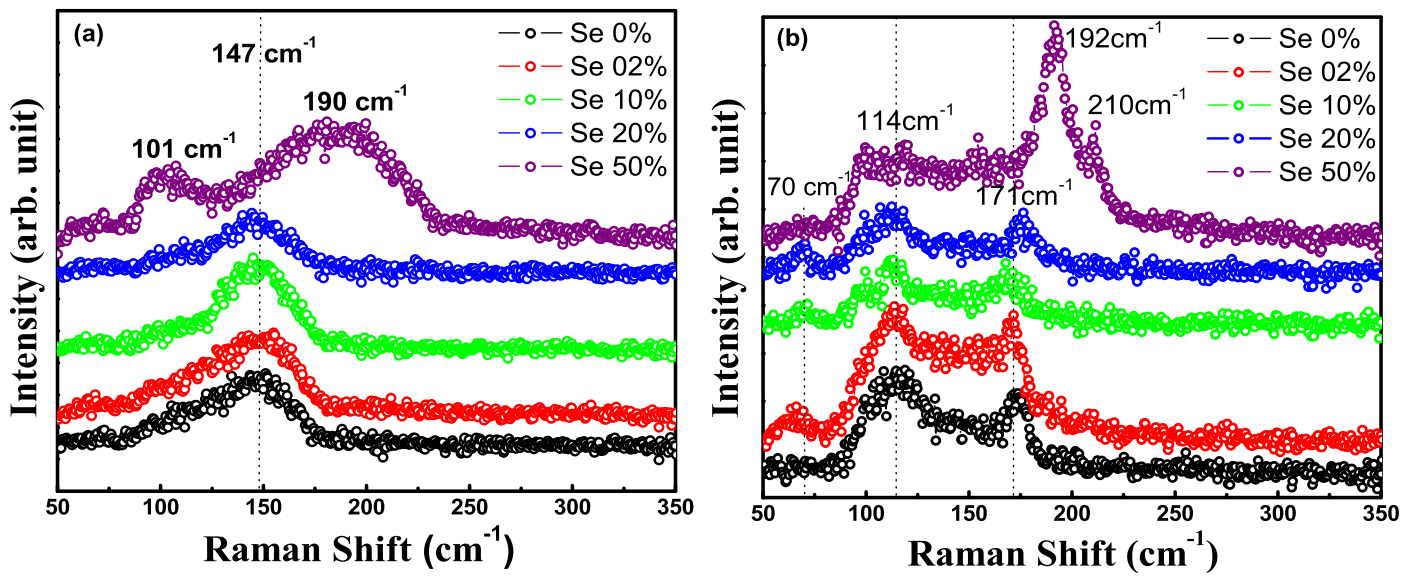

Figure $4 \mid$ Raman spectra of $(\mathrm{GST})_{1-\mathrm{x}} \mathrm{Se}_{\mathrm{x}}(\mathrm{a}) \mathrm{AD}$ and (b) annealed at $300^{\circ} \mathrm{C}$.

Band gap values of as deposited films varies from $0.63 \mathrm{eV}$ (GST alloy) to $1.05 \mathrm{eV}\left((\mathrm{GST})_{0.50} \mathrm{Se}_{0.50}\right.$ alloy). The widening of band gap in the amorphous phase is favorable to enhance the switching properties $^{34}$. The widening of band gap in amorphous materials has fundamental importance due to the presence of trap states, which plays an important role by filling up the charge carriers to become conductive in switching. A large band gap is required to have such trap states to exist in the amorphous phase ${ }^{34}$. For films annealed at $150^{\circ} \mathrm{C}$, the band gap reduced from the $\mathrm{AD}$ state which varies between $0.38 \mathrm{eV}$ (for GST) to $0.71 \mathrm{eV}$ (for $(\mathrm{GST})_{0.50} \mathrm{Se}_{0.50}$ ). At $300^{\circ} \mathrm{C}$, the band gap further reduces and varies between $0.24 \mathrm{eV}$ (for GST) to $0.53 \mathrm{eV}$ (for $(\mathrm{GST})_{0.50} \mathrm{Se}_{0.50}$ ).

The optical band gap increases with the increase of Se as shown in Fig. 6(a). When the films are annealed, the band gap is found to decrease (Fig. 6(b)). The increase in band gap indicates that more energy is required for conduction to occur. In $\mathrm{Ge}_{10} \mathrm{Se}_{90-\mathrm{x}} \mathrm{Te}_{\mathrm{x}}$, the band gap decreases with the increase of Te. The added Te substitutes Se which decreases the average cohesive energy and electronegativity $^{35}$. In the present case, the added Se might increase the cohesive energy and electronegativity by forming higher energy bonds (like $\mathrm{Ge}-\mathrm{Se}$ ) which results in the increase of band gap.

$\mathrm{A}$ higher value of $\mathrm{B}^{1 / 2}$ indicates less disorder ${ }^{36} . \mathrm{B}^{1 / 2}$ increases with annealing temperature indicating a decrease in disorder. During phase transition this defects/disorder decreases and an ordered arrangement of crystalline $\mathrm{NaCl} /$ hexagonal happens. Even in $\mathrm{NaCl}$ crystalline GST, $20 \%$ of cation sites in the Ge/Sb sub lattice are vacant and act as disorder in the system but it is less than the defects present in the amorphous phase. The addition of Se to $\mathrm{Ge}-\mathrm{Sb}-\mathrm{Te}$ induces structural changes in the system and increases the disorder in the amorphous state.
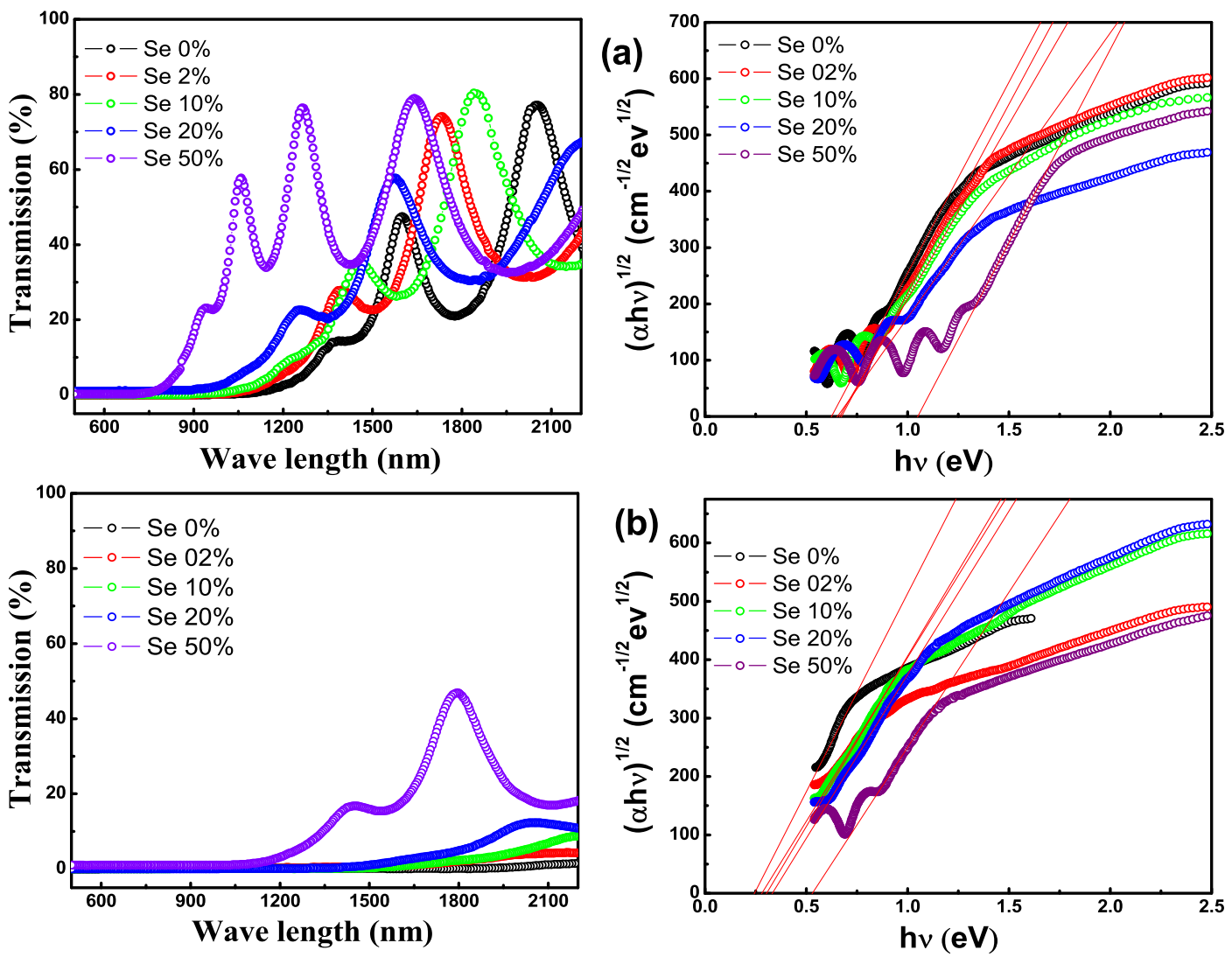

Figure $5 \mid$ Transmission spectra and the Tauc plot used for calculating the band gap of (a) AD and (b) annealed (at $\left.300^{\circ} \mathrm{C}\right)(\mathrm{GST})_{1-\mathrm{x}} \mathrm{Se}_{\mathrm{x}}$ films. 


\begin{tabular}{|c|c|c|}
\hline Sample & Band gap (eV) & $\mathrm{B}^{1 / 2}\left(\mathrm{~cm}^{-1 / 2} \mathrm{eV}^{-1 / 2}\right)$ \\
\hline GST AD & 0.63 & 678 \\
\hline$(G S T)_{0.98} \mathrm{Se}_{0.02} \mathrm{AD}$ & 0.67 & 671 \\
\hline$(G S T)_{0.90} \mathrm{Se}_{0.10} \mathrm{AD}$ & 0.66 & 621 \\
\hline$(G S T)_{0.80} \mathrm{Se}_{0.20} \mathrm{AD}$ & 0.64 & 492 \\
\hline$(G S T)_{0.50} S_{0.50} A D$ & 1.05 & 500 \\
\hline GST $150^{\circ} \mathrm{C}$ & 0.38 & 699 \\
\hline$(G S T)_{0.98} \mathrm{Se}_{0.02} 150^{\circ} \mathrm{C}$ & 0.47 & 680 \\
\hline$(G S T)_{0.90} \mathrm{Se}_{0.10} 150^{\circ} \mathrm{C}$ & 0.50 & 646 \\
\hline$(G S T)_{0.80} \mathrm{Se}_{0.20} 150^{\circ} \mathrm{C}$ & 0.57 & 611 \\
\hline$(G S T)_{0.50} \mathrm{Se}_{0.50} 150^{\circ} \mathrm{C}$ & 0.71 & 520 \\
\hline GST $300^{\circ} \mathrm{C}$ & 0.24 & 700 \\
\hline$(G S T)_{0.98} \mathrm{Se}_{0.02} 300^{\circ} \mathrm{C}$ & 0.28 & 683 \\
\hline $0.90 \mathrm{Se}_{0.10} 300^{\circ} \mathrm{C}$ & 0.30 & 661 \\
\hline $0.80 \mathrm{Se}_{0.20} 300^{\circ} \mathrm{C}$ & 0.33 & 685 \\
\hline $0.50 \mathrm{Se}_{0.50} 300^{\circ} \mathrm{C}$ & 0.53 & 531 \\
\hline
\end{tabular}

The addition Se in general increases the resistance of the as deposited films ${ }^{37}$. The change in resistance of $(\mathrm{GST})_{1-\mathrm{x}} \mathrm{Se}_{\mathrm{x}}$ films with annealing temperature are shown in Fig. 7. The resistance of the GST films shows a sudden drop when annealed at $100^{\circ} \mathrm{C}$. The discontinuity in resistance occurs at higher annealing temperatures for Se doped films. For the (GST) $)_{0.98} \mathrm{Se}_{0.02}$ and (GST) $)_{0.90} \mathrm{Se}_{0.10}$ films the drop in resistance is observed at $125^{\circ} \mathrm{C}$. The $(\mathrm{GST})_{0.80} \mathrm{Se}_{0.20}$ film shows the drop in resistance when annealed at $200^{\circ} \mathrm{C}$. The change in resistance is about 5 orders of magnitude. Annealing does not have much effect on $(\mathrm{GST})_{0.50} \mathrm{Se}_{0.50}$ film and change in resistance is not appreciable. The temperature dependence of resistance showed two transitions at $150^{\circ} \mathrm{C}$ and $240^{\circ} \mathrm{C}$ for GST and a single transition for $(\mathrm{GST})_{0.90} \mathrm{Se}_{0.10}$ at about $200^{\circ} \mathrm{C}$ (see the inset of Fig. 7.) ${ }^{38}$. This is a clear evidence for increased transition temperatures and thermal stability. The in-situ resistance measurements while cooling also showed a semiconducting nature ${ }^{38}$.

The stronger covalent bonds due to Se doping may reduce the atomic diffusivity and hence the $\mathrm{T}_{c}$ increases for Se doped GST films ${ }^{39}$. In general, the crystallization leads to an increase in grain size and roughness ${ }^{9,40}$. For the annealed film, an increase in grain size will lead to higher conductivity due to less electron scattering at the grain boundaries. The crystallization starts initially at the free surface of the film and grows slowly and islands of crystallites nucleate homogeneously all over the film. This lead to bigger grain size formation and higher conductivity as it crystallizes.

The write current can be reduced if the electrical resistivity of the GST film in the crystalline state is increased ${ }^{39}$. A higher $\mathrm{T}_{\mathrm{c}}$ increases the archival life time and maximum operation temperature. In present studies the Se doped GST shows a larger resistivity and increased

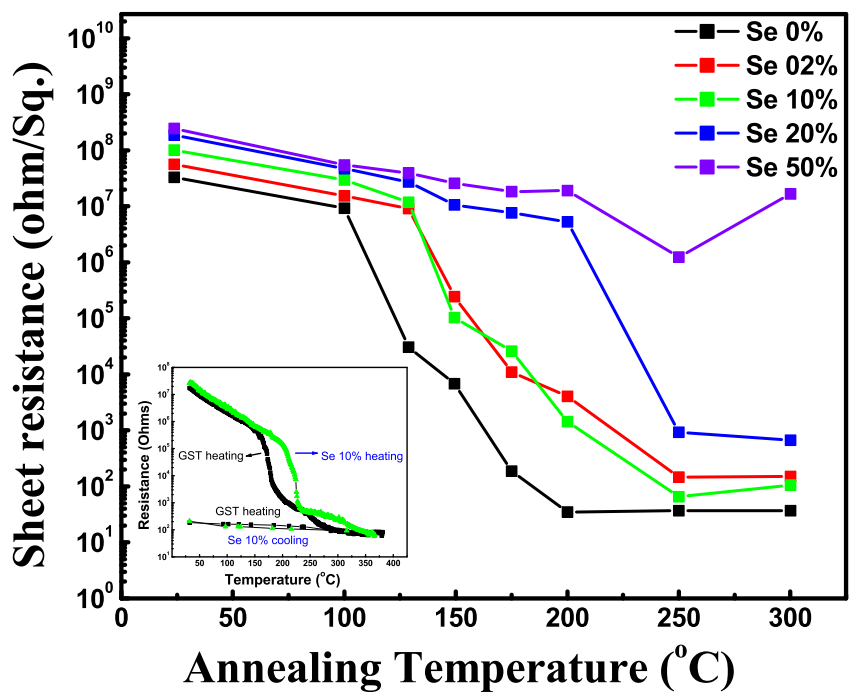

Figure $7 \mid$ Resistances of $(\mathrm{GST})_{1-\mathrm{x}} \mathrm{Se}_{\mathrm{x}}$ measured after isothermal annealing at different temperatures.

crystallization temperature for the crystalline phase which can significantly reduce the write current and can improve the retention time of the amorphous phase.

A simple device is designed and fabricated for comparing switching studies of various Se doped GST alloys. The Agilent Device Analyzer B1500A with tungsten probes was used to sweep the current and to simultaneously measure the voltage. Though the device area was large, this could give indicative (comparative) switching behaviors of undoped and Se doped GST alloys for further advanced studies.

The switching behavior of (GST) $)_{1-\mathrm{x}} \mathrm{Se}_{\mathrm{x}}$ films exhibiting memory switching are shown in Fig. 8. The highest Se content film $\left((\mathrm{GST})_{0.50} \mathrm{Se}_{0.50}\right)$ does not switch under high fields. The un-doped GST switched at a threshold voltage of $3.5 \mathrm{~V}$. The Se doped GST films switched at higher threshold voltages. For $(\mathrm{GST})_{0.98} \mathrm{Se}_{0.02}$, $(\mathrm{GST})_{0.90} \mathrm{Se}_{0.10}$ and $(\mathrm{GST})_{0.80} \mathrm{Se}_{0.20}$ films switching occurs at 4.4, 4.5 and $5.7 \mathrm{~V}$ respectively. The added Se might preferably bond with Ge and form a Ge-Se phase ${ }^{30}$. This Ge-Se phase may have higher resistance which might push the threshold voltage to higher side. It is seen in the XRD studies that the crystallization is retarded by the Se presence and therefore the activation energy for switching. It is reported that in Si doped GST, the threshold voltage increases with Si addition ${ }^{39}$. It is found that the threshold voltage is reducing linearly with electrode separation ${ }^{41}$. So there is a possibility of reducing the set and reset voltage by reducing the volume of $(\mathrm{GST})_{1-\mathrm{x}} \mathrm{Se}_{\mathrm{x}}$ device.
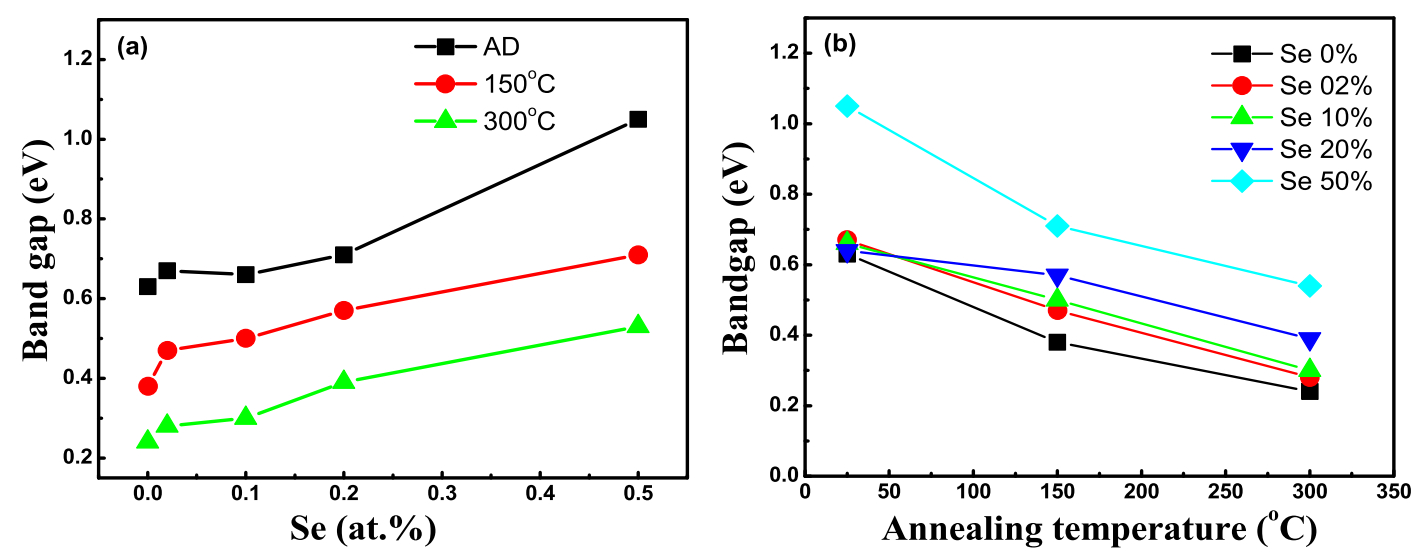

Figure $6 \mid$ (a) Variation of the band gap with Se content and (b) with annealing temperatures. 


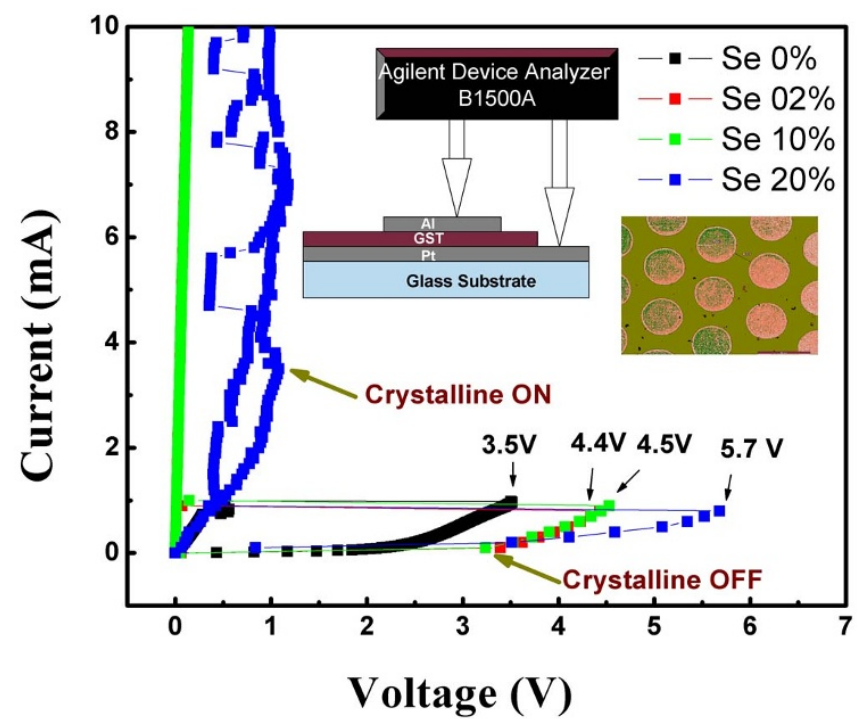

Figure 8 I-V switching studies of as deposited (GST) ${ }_{1-\mathrm{x}} \mathrm{Se}_{\mathrm{x}}$ films. Inset show the device micrograph and schematic of the device fabricated.

\section{Conclusions}

The effect Se doping upon the phase change properties of GST has been studied. All the as deposited $(\mathrm{GST})_{1-\mathrm{x}} \mathrm{Se}_{\mathrm{x}}$ films are amorphous. The $(\mathrm{GST})_{1-\mathrm{x}} \mathrm{Se}_{\mathrm{x}}$ films annealed at $150^{\circ} \mathrm{C}$ are crystallized in $\mathrm{NaCl}$ structure for $\mathrm{x} \leq 0.02$. The films with $0.10 \leq \mathrm{x} \leq 0.20$ showed direct transition to hexagonal structure and the intermediate $\mathrm{NaCl}$ structure is not observed. An extra Te-Te or Te-Se layer and filling up of the vacancies in the $\mathrm{NaCl}$ structure of GST can possibly drive the system directly to the hexagonal structure. Resistance and thermal stability increased with the increase of Se. The overall bond energy of the system increases due to the formation $\mathrm{Ge}-\mathrm{Se}$ bonds which increases the $T_{c}$. The larger resistivity of the crystalline phase in a PCM cell will be beneficial as it reduces the write current. The band gap and disorder increases with Se addition in the as deposited amorphous film and decreases in the annealed crystalline films. Band gap values of the as deposited films vary from 0.63 to $1.05 \mathrm{eV}$. The resistance contrast between the amorphous and the crystalline phases is more than 5 orders of magnitude compared to GST. Similarly, higher $T_{c}$ found in these alloys is advantageous for multiple memory storage applications. These findings show that the band gap, threshold voltage and transition temperatures of GST alloys can be tuned by the addition of Se. Importantly, this study showed that the general understanding of the requirement of the metastable $\mathrm{NaCl}$-like structure for the feasible phase change properties may not be correct and the direct amorphous to stable hexagonal structural transition also gives the similar kind of switching. However, crystallization kinetics of amorphous to hexagonal transition need to be studied to understand the switching speed of the Se doped GST alloys.

\section{Methods}

Sample Preparation. Thin film samples of $(\mathrm{GST})_{1-\mathrm{x}} \mathrm{Se}_{\mathrm{x}}(\mathrm{x}=0,0.02,0.10,0.20$ and 0.50 at.\%) were deposited from their bulk alloys on to glass substrates by thermal evaporation method.

Characterizations. Thickness of the films measured with a Stylus profiler (DEKTAK VEECO), was found to be $500 \mathrm{~nm}$. The compositions of the deposited films were verified with Energy-dispersive X-ray spectroscopy. Films were annealed at selected temperatures for $1 \mathrm{~h}$ at a vacuum of $10^{-5}$ mbar using a resistive heater in a vacuum chamber. The annealed films were subjected to X-ray diffraction (XRD) to identify the crystalline structures with $\mathrm{Cu}_{\mathrm{K} \alpha}$ radiation $(\lambda=1.54 \AA)$ in a BRUKER D8 ADVANCE diffractometer. Transmission spectra of the films were taken in the VISNIR range (550-2200 nm) using PerkinElmer Lambda 750 VIS-NIR Spectrometer. Raman spectroscopy (LabRAM HR system) measurements were performed using $514.5 \mathrm{~nm}$ Argon laser with a charge coupled device detector in backscattering geometry with a spectral resolution of $0.5 \mathrm{~cm}^{-1}$. The power with the filter is $0.01 \mathrm{~mW}$ on the sample and the accumulation time for each spectrum is 180 seconds, which did not induce any crystallization. Various laser power-filter combinations have been tried to reduce the laser induced crystallisation on the sample surface. Filter D0.3 with laser set power represents power/2, filter D1 gives power/10 and filter D2 gives power/ 100. Electrical switching studies have been performed using a probe station with Agilent Device Analyzer B1500A. The active area of the device was $376 \mu^{2}$ in a vertical sandwich geometry of Pt $(100 \mathrm{~nm}) / \mathrm{PCM}(500 \mathrm{~nm}) / \mathrm{Al}(300 \mathrm{~nm})$ on glass substrates. Current was swept between upper Al electrode and lower Pt electrodes and the corresponding voltages were measured. Two probe resistance measurements were carried out using the same Agilent Device Analyzer on the samples annealed at different temperatures. Each reading is an average of three measurements.

1. Shportko, K. et al. Resonant bonding in crystalline phase-change materials. Nat. Mater. 7, 65-658 (2008).

2. Ovshinsky, S. R. Reversible Electrical Switching Phenomena in Disordered Structures, Physical Review Letters. Phys. Rev. Lett. 21, 1450 (1968).

3. Tomforde, J. et al. The influence of Se doping upon the phase change characteristics of $\mathrm{GeSb}_{2} \mathrm{Te}_{4}$, Solid State Sciences. Solid State Sci. 11, 683-687 (2009).

4. Matsunaga, T., Yamada, N. \& Kubota, Y. Structures of stable and metastable $\mathrm{Ge}_{2} \mathrm{Sb}_{2} \mathrm{Te}_{5}$, an intermetallic compound in $\mathrm{GeTe}-\mathrm{Sb}_{2} \mathrm{Te}_{3}$ pseudobinary systems. Acta Crystallogr., Sect. B. 60, 685-691 (2004).

5. Lu, Y. et al. Ga-Sb-Se material for low-power phase change memory. Appl. Phys. Lett. 99, 243111 (2011).

6. Yoon, S.-M. et al. Sb-Se-based phase-change memory device with lower power and higher speed operations. IEEE Electron Device L. 27, 445-447 (2006).

7. Friedrich, I., Weidenhof, V., Njoroge, W., Franz, P. \& Wuttig, M. Structural transformations of $\mathrm{Ge}_{2} \mathrm{Sb}_{2} \mathrm{Te}_{5}$ films studied by electrical resistance measurements. J. Appl. Phys. 87, 4130 (2000).

8. Mendoza-Galván, A. \& González-Hernández, J. Drude-like behavior of Ge:Sb:Te alloys in the infrared. J. Appl. Phys. 87, 760 (2000).

9. Song, K. H., Kim, S. W., Seo, J. H. \& Lee, H. Y. Characteristics of amorphous $\mathrm{Ag}_{0.1}\left(\mathrm{Ge}_{2} \mathrm{Sb}_{2} \mathrm{Te}_{5}\right)_{0.9}$ thin film and its ultrafast crystallization. J. Appl. Phys. 104, $103516(2008)$.

10. Yamada, N. \& Matsunaga, T. Structure of laser-crystallized $\mathrm{Ge}_{2} \mathrm{Sb}_{2} \mathrm{Te}_{5}$ sputtered thin films for use in optical memory. J. Appl. Phys. 88, 7020 (2000).

11. Kolobov, A. V. et al. Understanding the phase-change mechanism of rewritable optical media. Nat. Mater. 3, 703-708 (2004).

12. Sun, Z., Zhou, J. \& Ahuja, R. Structure of Phase Change Materials for Data Storage. Phys. Rev. Lett. 96, 055507 (2006).

13. Vinod, E. M., Naik, R., Ganesan, R. \& Sangunni, K. S. Signatures of $\mathrm{Ge}_{2} \mathrm{Sb}_{2} \mathrm{Te}_{5}$ film at structural transitions. J. Non-Cryst. Solids 358, 2927-2930 (2012).

14. Privitera, S. et al. Crystallization and phase separation in $\mathrm{Ge}_{2+\mathrm{x}} \mathrm{Sb}_{2} \mathrm{Te}_{5}$ thin films. J. Appl. Phys. 94, 4409 (2003).

15. Wang, K., Steimer, C., Wamwangi, D., Ziegler, S. \& Wuttig, M. Effect of indium doping on $\mathrm{Ge}_{2} \mathrm{Sb}_{2} \mathrm{Te}_{5}$ thin films for phase-change optical storage. Appl. Phys. A 80, 1611-1616 (2005).

16. Phillips, J. C. \& Thorpe, M. F. Constraint theory, vector percolation and glass formation. Solid State Commun. 53, 699-702 (1985).

17. Narayanan, R. A., Asokan, S. \& Kumar, A. Evidence concerning the effect of topology on electrical switching in chalcogenide network glasses. Phys. Rev. B. 54, 4413 (1996).

18. Song, S. A., Zhang, W., Sik Jeong, H., Kim, J.-G. \& Kim, Y.-J. In situ dynamic HRTEM and EELS study on phase transitions of $\mathrm{Ge}_{2} \mathrm{Sb}_{2} \mathrm{Te}_{5}$ chalcogenides. Ultramicroscopy 108, 1408-1419 (2008).

19. Yamada, N. Origin, secret, and application of the ideal phase-change material GeSbTe. Phys. Status Solidi B. 249, 1837-1842 (2012).

20. Cho, E., Yoon, S., Yoon, H. R. \& Jo, W. Micro-Raman Scattering Studies of Ge-SbTe Bulk Crystals and Nanoparticles. J. Korean Phys. Soc. 48, 1616-1619 (2006).

21. Liu Bo, Zhi-Tang, S., Ting, Z., Song-Lin, F. \& Bomy, C. Raman spectra and XPS studies of phase changes in $\mathrm{Ge}_{2} \mathrm{Sb}_{2} \mathrm{Te}_{5}$ films. Chinese Phys. 13, 1947-1950 (2004)

22. Andrikopoulos, K. S., Yannopoulos, S. N., Kolobov, A. V., Fons, P. \& Tominaga, J Raman scattering study of $\mathrm{GeTe}$ and $\mathrm{Ge}_{2} \mathrm{Sb}_{2} \mathrm{Te}_{5}$ phase-change materials. J. Phys. Chem. Solids 68, 1074-1078 (2007).

23. Nemec, P. et al. Ge-Sb-Te thin films deposited by pulsed laser: An ellipsometry and Raman scattering spectroscopy study. J. Appl. Phys. 106, 103509 (2009).

24. Tominaga, J. \& Atoda, N. Study of the Crystallization of GeSbTe Films by Raman Spectroscopy. Jpn. J. Appl. Phys. 38, L322-L323 (1999).

25. Sosso, G. C., Caravati, S., Mazzarello, R. \& Bernasconi, M. Raman spectra of cubic and amorphous $\mathrm{Ge}_{2} \mathrm{Sb}_{2} \mathrm{Te}_{5}$ from first principles. Phys. Rev. B. 83, 134201 (2011)

26. De Bastiani, R. et al. Crystallization of ion amorphized $\mathrm{Ge}_{2} \mathrm{Sb}_{2} \mathrm{Te}_{5}$ thin films in presence of cubic or hexagonal phase. J. Appl. Phys. 107, 113521 (2010).

27. Sergey, K., Kudoyarova, V., Nguyen, H. P., Smirnov, A. \& Lebedev, V. Influence of doping on the structure and optical characteristics of $\mathrm{Ge}_{2} \mathrm{Sb}_{2} \mathrm{Te}_{5}$ amorphous films. phys. status solidi (c) 8, 2688-2691 (2011).

28. Tronc, P., Bensoussan, M., Brenac, A. \& Sebenne, C. Optical-Absorption Edge and Raman Scattering in $\mathrm{Ge}_{\mathrm{x}} \mathrm{Se}_{1-\mathrm{x}}$ Glasses. Phys. Rev. B. 8, 5947 (1973).

29. Sugai, S. Stochastic random network model in Ge and Si chalcogenide glasses. Phys. Rev. B. 35, 1345 (1987) 
30. Vinod, E. M., Singh, A. K., Ganesan, R. \& Sangunni, K. S. Effect of selenium addition on the GeTe phase change memory alloys. J. Alloys Compd. 537, 127-132 (2012).

31. Tauc, J., Grigorov, R. \& Vancu, A. Optical properties and electronic structure of amorphous Germanium. Phys. Status Solidi 15, 627-637 (1966).

32. Davis, E. A. \& Mott, N. F. Conduction in non-crystalline systems V. Conductivity, optical absorption and photoconductivity in amorphous semiconductors. Philos. Mag. 22, 903-922 (1970).

33. Vinod, E. M., Naik, R., Faiyas, A. P. A., Ganesan, R. \& Sangunni, K. S. Temperature dependent optical constants of amorphous $\mathrm{Ge}_{2} \mathrm{Sb}_{2} \mathrm{Te}_{5}$ thin films. J. Non-Cryst. Solids 356, 2172-2174 (2010).

34. Welnic, W. et al. Unravelling the interplay of local structure and physical properties in phase-change materials. Nat. Mater 5, 56-62 (2006).

35. Sharma, P. \& Katyal, S. C. Effect of tellurium addition on the physical properties of germanium selenide glassy semiconductors. Physica B: Condensed Matter 403, 3667-3671(2008).

36. Zanatta, A. R. \& Chambouleyron, I. Absorption edge, band tails, and disorder of amorphous semiconductors. Phys. Rev. B. 53, 3833 (1996).

37. Vinod, E. M. \& Sangunni, K. S. The effect of Se doping on spectroscopic and electrical properties of GeTe. Thin Solid Films 550, 569-574 (2014).

38. Vinod, E. M., Ramesh, K., Ganesan, R. \& Sangunni, K. S. Direct hexagonal transition of amorphous $\left(\mathrm{Ge}_{2} \mathrm{Sb}_{2} \mathrm{Te}_{5}\right)_{0.9} \mathrm{Se}_{0.1}$ thin films. Appl. Phys. Lett. 104, 063505 (2014)

39. Bao-Wei, Q. et al. Improvement of Electrical Properties of the $\mathrm{Ge}_{2} \mathrm{Sb}_{2} \mathrm{Te}_{5}$ Film by Doping Si for Phase Change Random Access Memory. Chinese Phys. Lett. 23, 172 (2006).

40. Shin, M.-J. et al. Chemical Bonding Characteristics of $\mathrm{Ge}_{2} \mathrm{Sb}_{2} \mathrm{Te}_{5}$ for Thin Films. J. Korean Phys. Soc. 44, 10-13 (2004).
41. Xiong, F., Liao, A. D., Estrada, D. \& Pop, E. Low-Power Switching of PhaseChange Materials with Carbon Nanotube Electrodes. Science 332, 568-570 (2011).

\section{Acknowledgments}

The authors thank Department of Science and Technology, India for the financial support and Micro and Nano Characterization Facility (MNCF) at the Centre for Nano Science and Engineering (CeNSE), Indian Institute of Science, Bangalore, India.

\section{Author contributions}

E.M.V. conducted the experiments and wrote the main manuscript text under K.S.S. guidance. K.R. edited and suggested some modifications. All authors reviewed the manuscript.

\section{Additional information}

Competing financial interests: The authors declare no competing financial interests.

How to cite this article: Vinod, E.M., Ramesh, K. \& Sangunni, K.S. Structural transition and enhanced phase transition properties of Se doped $\mathrm{Ge}_{2} \mathrm{Sb}_{2} \mathrm{Te}_{5}$ alloys. Sci. Rep. 5, 8050; DOI:10.1038/srep08050 (2015)

This work is licensed under a Creative Commons Attribution-NonCommercialNoDerivs 4.0 International License. The images or other third party material in this article are included in the article's Creative Commons license, unless indicated otherwise in the credit line; if the material is not included under the Creative Commons license, users will need to obtain permission from the license holder in order to reproduce the material. To view a copy of this license, visit http:// creativecommons.org/licenses/by-nc-nd/4.0/ 